\title{
Cardiorenal disease in the United States: Future health care burden and potential impact of novel therapies
}

\author{
Phil McEwan, PhD; Angharad R Morgan, PhD; Rebecca Boyce, MSc; Natalie Green, PhD; \\ Bruce Song, BS; Joanna Huang, PharmD; Klas Bergenheim, PhD
}

\section{What is already known about this subject}

- Type 2 diabetes, heart failure, and chronic kidney disease often occur together (cardiorenal disease), as a consequence of shared risk factors as well as the bidirectional effect between the heart and kidneys with dysfunction in one organ promoting dysfunction in the other organ.

- Sodium-glucose cotransporter 2 inhibitors (SGLT2is), although first developed as glucose-lowering therapies for the treatment of diabetes, have demonstrated significant outcome benefits across the cardiorenal continuum.

\author{
What this study adds \\ - Cardiorenal disease is likely to inflict \\ a significant burden on health care \\ systems over the next 10 years. \\ - The use of SGLT2is to manage \\ cardiorenal patients from a holistic \\ perspective is likely to result in a \\ substantial reduction in the number of \\ hospitalizations, disease prevalence, \\ and associated costs over the next \\ decade.
}

\section{Author affiliations}

Phil McEwan, PhD; Angharad R Morgan, PhD; and Rebecca Boyce, MSc, Health Economics and Outcomes Research Ltd, Cardiff, United Kingdom. Natalie Green, PhD, Huron Consulting Group, London, United Kingdom. Bruce Song, BS, and Joanna Huang, PharmD, Global Pricing and Market Access, AstraZeneca, Hockessin, DE. Klas Bergenheim, PhD, BioPharmaceuticals R \& D, AstraZeneca, Gothenburg, Sweden.

AUTHOR CORRESPONDENCE:

Phil McEwan, phil.mcewan@heor.co.uk

J Manag Care Spec Pharm. 2022;28(4):415-24

Copyright $\odot 2022$, Academy of Managed Care Pharmacy. All rights reserved.

RESULTS: The model estimates that total prevalence across all disease states will have increased by $28 \%$ in 2030 . Cumulatively, the direct health care cost of cardiorenal disease between 2021 and 2030 is estimated at $\$ 4.8$ trillion. However, treatment with dapagliflozin has the potential to reduce disease prevalence by $8.0 \%$ and estimated cumulative service delivery costs by $3.6 \%$ by 2030 .

CONCLUSIONS: Considering a holistic approach when managing patients with cardiorenal disease offers an opportunity to reduce the disease burden over the next 10 years in the US population. dence describing the burden of those diagnosed with T2DM, HF, and CKD in the
United States as individual discrete chronic economic impact of novel therapies in this population.

Me developed a compartmental with an annual time cycle population period 2021-2030 (either in isolation or combined). The model was used to explore the potential impact of novel therapies such as sodium-glucose cotransporter 2 inhibitors on future disease burden, by extrapolating the results of relevant clinical trials to representative patient populations. 
The concept of value for money, maximizing health outcomes per dollar spent, is an important consideration in health care decision making. The acquisition cost of new technologies is routinely justified using a cost-effectiveness analyses (CEA) framework. ${ }^{1}$ CEA seeks to estimate the relationship between cost and health outcomes associated with competing interventions; in chronic diseases this will typically involve the evaluation of therapies, at specific points in the clinical pathway, balancing lifetime therapy costs with the potential for reducing complication costs and increasing quality-adjusted life-years. CEA is both an established and widely used framework offering insight into "value for money" with reference to an average cohort profile, evaluated at a specific point in the disease continuum with a focus on quantifying the value of competing technologies. Although it is necessary for a CEA to incorporate all relevant costs and resource utilization associated with disease management, it is not required to consider whether these resources are available; therefore, although the cost of hospital bed days, health care professionals, and ambulatory care are considered, for example, their availability is not. Consequently, a CEA informs "value for money" decision making from the perspective of pharmacy spending but misses the opportunity to communicate the implications upon health care system capacity associated with disease management within a dynamic prevalent population.

This is of particular relevance to cardiorenal disease, comprised of conditions that involve both the heart and/ or kidneys, where the acute or chronic dysfunction in one organ may induce acute or chronic dysfunction in the other. ${ }^{2}$ The economic burden of heart failure (HF) and chronic kidney disease (CKD) in the United States is considerable. The direct health cost of HF has been projected to increase from $\$ 30.7$ billion in 2012 to $\$ 69.8$ billion in 2030, ${ }^{3}$ whereas the combined Medicare spend on CKD and endstage renal disease (ESRD) is currently estimated at more than $\$ 120$ billion per year. ${ }^{4}$ The established bidirectional link between renal pathology and HF makes this even more significant within the context of existing cardiovascular risk factors, such as type 2 diabetes mellitus (T2DM). There is a negative synergistic effect of HF, renal insufficiency, or worsening renal function in T2DM that increases the likelihood of hospitalization, rehospitalization, and in-hospital outcomes, such as mortality and increased length of stay. Consequently, the service delivery implications of managing cardiorenal disease are substantial.

Sodium-glucose cotransporter 2 inhibitors (SGLT2is), although first developed as glucose-lowering therapies for the treatment of diabetes, have demonstrated significant outcome benefits across the cardiorenal continuum in a series of landmark HF and CKD outcome trials in people with and without diabetes, such that a class of drugs initially developed for the management of 1 chronic condition has migrated across multiple therapeutic indications and chronic disease areas. This introduces an important challenge for considering how to estimate the value of an SGLT2i in clinical practice. Typically, this would be undertaken within the context of a specific indication, eg, as a treatment for T2DM, HF, or CKD, using a CEA framework. Indeed, SGLT2is have been found to be cost-effective for the management of T2DM, ${ }^{5,6}$ as well as for the management of patients with $\mathrm{HF}$, with and without $\mathrm{T} 2 \mathrm{DM},{ }^{7}$ and in those with CKD, with and without T2DM. ${ }^{8}$ However, as T2DM, $\mathrm{CKD}$, and HF are closely linked in terms of pathophysiology and are integrally associated with one another within the context of health care system activity, CEA of the individual diseases may miss concomitant benefits on related diseases. Furthermore, as previously highlighted, due consideration to system capacity is lacking within a conventional CEA. There is, therefore, merit in considering a more holistic system perspective when undertaking an economic evaluation of therapeutic and technologic innovation within the context of health care delivery. Thus, the objective of this study was 2-fold: first, to estimate the current and future economic burden of T2DM, HF, and CKD (both individually and combined) using established epidemiologic, cost, and resource utilization evidence and, second, to evaluate the potential impact of novel pharmacologic therapies in cardiorenal medicine from a holistic whole-disease perspective.

\section{Methods}

\section{OVERVIEW}

We developed a compartmental Markov model with an annual time cycle to model an evolving prevalent US patient population with T2DM, HF, or CKD over the period 20212030 (either in isolation or combined). Specifically, the model was designed to estimate the expected future prevalence of the following health states, taking into consideration disease incidence, an aging population, and increases in life expectancy: $\mathrm{T}^{2} \mathrm{DM}_{0}$ (no CKD or HF), $\mathrm{CKD}_{0}$ (no T2DM or HF), $\mathrm{HF}_{0}$ (no T2DM or CKD), T2DM+HF (no CKD), T2DM+CKD (no HF), $\mathrm{HF}+\mathrm{CKD}$ (no T2DM), and T2DM+HF+CKD.

Targeted peer-reviewed data characterizing diseasespecific resource utilization relating to hospital bed days, emergency department, intensive care, outpatient, nurse visits, physician office visits, home visits, dialysis sessions, and transplantations were applied to the disease prevalence projections. 


\section{TABLE 1 Summary of Treatment Effects Across Clinical Trials (Hazard Ratios) and the Representativeness of Each Trial to the General Population}

\begin{tabular}{|c|c|c|c|c|c|c|c|c|}
\hline \multirow[b]{2}{*}{ Therapy } & \multirow[b]{2}{*}{ Clinical trial } & \multirow[b]{2}{*}{$\begin{array}{l}\text { Clinical } \\
\text { indication }\end{array}$} & \multicolumn{4}{|c|}{ Hazard ratios } & \multicolumn{2}{|c|}{ Trial representativeness } \\
\hline & & & $\begin{array}{c}\text { HF } \\
\text { incidence }\end{array}$ & HHF & $\begin{array}{c}\text { CKD } \\
\text { incidence }\end{array}$ & $\begin{array}{c}\text { ESRD } \\
\text { incidence }\end{array}$ & $\begin{array}{c}\text { Population, } \\
\%\end{array}$ & Source \\
\hline \multirow{3}{*}{ Dapagliflozin } & DECLARE $^{11,14}$ & T2DM & 0.73 & & 0.54 & 0.31 & 39.8 & Wittbrodt et al $2018^{15}$ \\
\hline & DAPA-CKD ${ }^{42}$ & CKD & 0.51 & & 0.53 & 0.64 & 11.5 & $\underline{\text { Supplementary Tables 5A-D }}$ \\
\hline & DAPA-HF ${ }^{43}$ & $\mathrm{HF}$ & & 0.70 & & & 50.0 & Assumed HFrEF population \\
\hline \multirow{2}{*}{ Empagliflozin } & EMPA-REG ${ }^{12,44}$ & T2DM & 0.65 & & 0.56 & 0.45 & 4.1 & Wittbrodt et al $2018^{41}$ \\
\hline & EMPEROR-Reduced ${ }^{45}$ & $\mathrm{HF}$ & & 0.69 & 0.50 & 0.50 & 50.0 & Assumed HFrEF population \\
\hline Canagliflozin & CANVAS ${ }^{13,46}$ & T2DM & 0.67 & & 0.60 & & 8.8 & Wittbrodt et al $2018^{41}$ \\
\hline Sacubitril \& valsartan & PARADIGM-HF 47 & $\mathrm{HF}$ & & 0.79 & & & 50.0 & Assumed HFrEF population \\
\hline
\end{tabular}

${ }^{a}$ Available in online article.

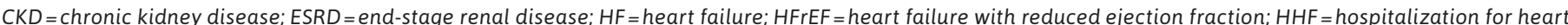
failure; T2DM = type 2 diabetes mellitus.

Finally, the model was used to explore the potential impact of selected therapies and their associated therapeutic profile on future disease burden, by extrapolating the results of relevant clinical trials to representative patient populations.

Disease prevalence predictions for each forecast year, for the period 2021-2030 and for each of the 7 health states above were calculated following a consistent approachadding the number of incident patients to survivors from the previous year, as follows:

$$
P_{x}(t+1)=P_{x}(t) \times\left(1-M_{x}\right)+I_{x}(t)(1)
$$

where $P_{x}(t)$ represents the prevalence of health state $x$ at time $t ; M_{x}$ represents the annual probability of mortality in health state $x$ and $I_{x}(t)$ is the annual incidence of health state $x$ at time $t$; where $x$ reflects health states 1 to 7 detailed above.

Due to the chronic nature of the diseases modeled, patients are not able to transition out of a health state once they have been diagnosed, unless by the incidence of another comorbidity or death. For example, once a patient has been diagnosed with T2DM, they are not able to transition back into the healthy population; however, they are subject to an annual probability of developing either HF or CKD and a state-specific probability of mortality every year. Within the model, incident CKD can be diagnosed at any CKD stage. Once diagnosed, patients progress through CKD stages at a constant annual rate. A full list of transition equations applied in the model are provided in Supplementary Table 1 (available in online article), and a conceptual schematic of the model is provided in the
Supplementary Figure 1 (available in online article), using notation defined in Supplementary Table 1.

\section{POPULATION INPUTS, COSTS, AND RESOURCE UTILIZATION}

Population level T2DM incidence and prevalence are modeled for 3 specific age groups (18-44, 45-64, and 65+ years) with HF stratified by age ( $<65 \mathrm{vs} \geq 65$ years), CKD, and diabetes status. CKD incidence, prevalence, and mortality were stratified by stage, diabetes, and HF status. Adjustments to disease specific prevalence was also undertaken to ensure only patients aware of their disease status were included in the analysis. Supplementary Table 2 lists the population level inputs applied in the model. Prevalence was calculated using a baseline adult population of 250,191,821 in $2020,{ }^{9}$ which was further stratified into relevant age groups to calculate the prevalence of T2DM and HF by age (Supplementary Table 2).

Costs were inflated to 2019 values using indices derived from the Bureau of Labor Statistics. ${ }^{10}$ For T2DM and HF, health-state-related costs are evaluated based on consumed health-state-specific resources attributed to each disease per year. For CKD patients, stage-specific costs attributed to CKD have been sourced and are applied to the prevalent population on an annual basis. Where patients have a diagnosis of 1 disease, they are assumed to incur the resource use and cost related to that single disease area. Where patients have more than 1 disease, resource use and costs are assumed to be applied additively, ie, a patient with T2DM and HF will incur the total resource use associated with T2DM, as well as the total resource use associated 


\section{FIGURE 1}
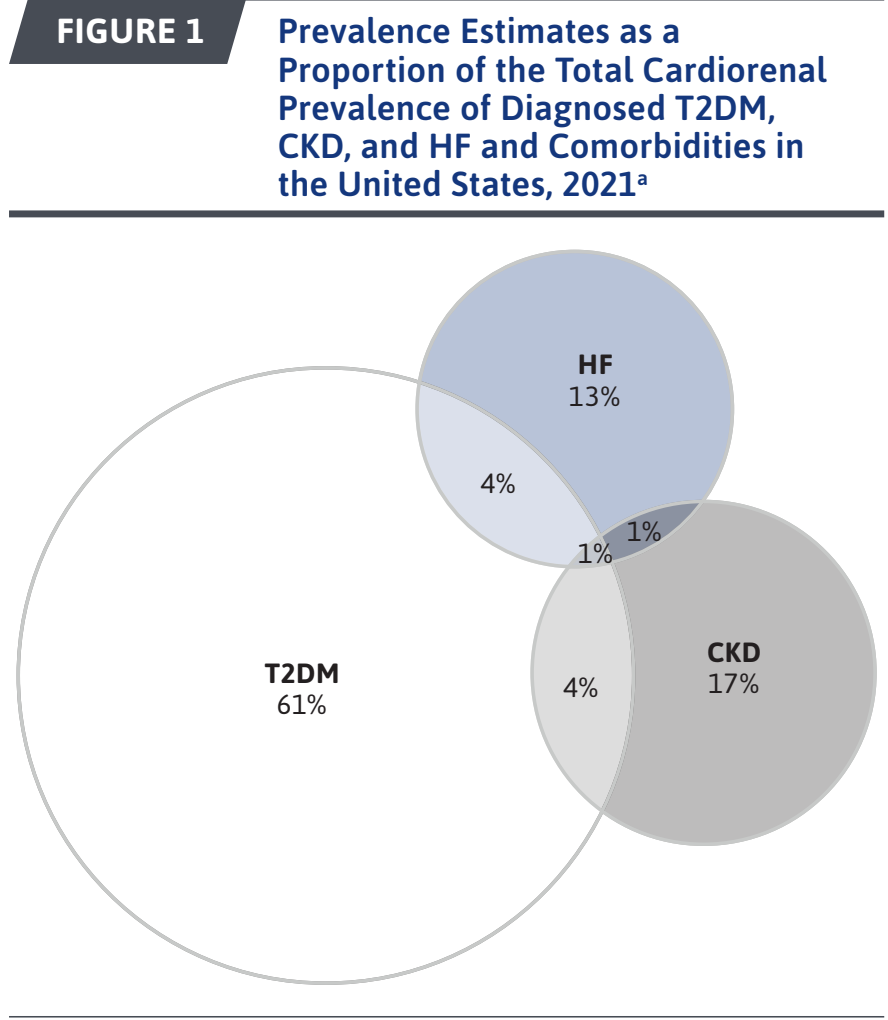

${ }^{a}$ Cardiorenal prevalence represents approximately $15 \%$ of the total adult population.

$C K D=$ chronic kidney disease; $H F=$ heart failure; $T 2 D M=$ type 2 diabetes mellitus.

with HF. No double counting is assumed to occur under this assumption as relevant resource use and cost inputs have been sourced as only those attributed to each disease area. Costs and resource utilization applied in the model are presented in Supplementary Tables 3 and 4 , respectively.

\section{EVALUATING THE POTENTIAL IMPACT OF NOVEL THERAPIES ON FUTURE CARDIORENAL DISEASE BURDEN}

Treatment effects were considered in terms of a reduction of hospitalization for heart failure (HHF) events, a reduced incidence of CKD and ESRD and a slower rate of estimated glomerular filtration rate decline in patients with prevalent CKD. Where treatment prevented an initial HHF event in patients with CKD or T2DM, this was assumed to prevent the incidence of HF in these patients. Cost benefits of treatment are captured in terms of cost savings from a reduced utilization of resource use due to events avoided. The direct effect of mortality benefits from treatments has not been considered in the analysis; however, due to a lower incidence of events and comorbid disease states following treatment initiation, there is an indirect benefit on mortality in the analysis.

The model considers the impact of treatment uptake for the following SGLT2 inhibitors: dapagliflozin (in all disease states), empagliflozin (in T2DM, HF+T2DM and $\mathrm{CKD}+\mathrm{T} 2 \mathrm{DM}$ ) and canagliflozin (in T2DM, HF+T2DM, CKD+T2DM). Furthermore, the potential impact of treatment uptake with sacubitril and valsartan in patients with HF was also evaluated.

The analytical approach undertaken was to first estimate disease prevalence and incidence using published evidence and then apply therapeutic benefits using trial reported hazard ratios (HRs) as presented in Table 1. In all treatment uptake scenarios, we restricted therapeutic benefit to those HF patients with reduced ejection fraction (HFrEF) only and those CKD patients at risk of progression (consistent with the trial populations). Table 1 also provides estimates of the generalizability of each respective trial indicating the proportion of the relevant diagnosed population to which a treatment effect is applied. Our overall analytical approach implies the control arms of the cardiovascular outcome trials, ${ }^{11-13}$ which included a combination of glucose-lowering therapy, antihypertensive therapy, lipid-lowering therapy, and anticoagulants, reflect current standard therapy. A detailed explanation of the methodology used to derive transition probabilities and their adjustment via the application of trial specific HRs is provided in Supplementary Methods.

Finally, in the United States negotiated discounts on pharmaceutical list prices are common, consequently the degree of incremental cost or cost saving represents a confidential variable. We have therefore sought to characterize the structural cost consequences associated with the application of therapy innovation setting aside acquisition cost, which are a requirement only in CEAs and, thereby, focusing on system-related costs. The cost-effectiveness of SGLT2is in T2DM in a US setting has been demonstrated elsewhere. $^{5}$

\section{Results}

\section{CURRENT AND FUTURE US BURDEN OF CARDIORENAL DISEASE}

Published literature regarding disease incidence and prevalence indicates an estimated 36 million patients will have been diagnosed in the United States with T2DM, HF, or CKD in 2021, reflecting approximately $15 \%$ of the total US adult population. Figure 1 presents a Venn diagram illustrating 


\section{FIGURE 2 Total Projected Number of Patients Estimated With Diagnosed T2DM, CKD, and HF and Comorbidities in the United States, 2021-2030}

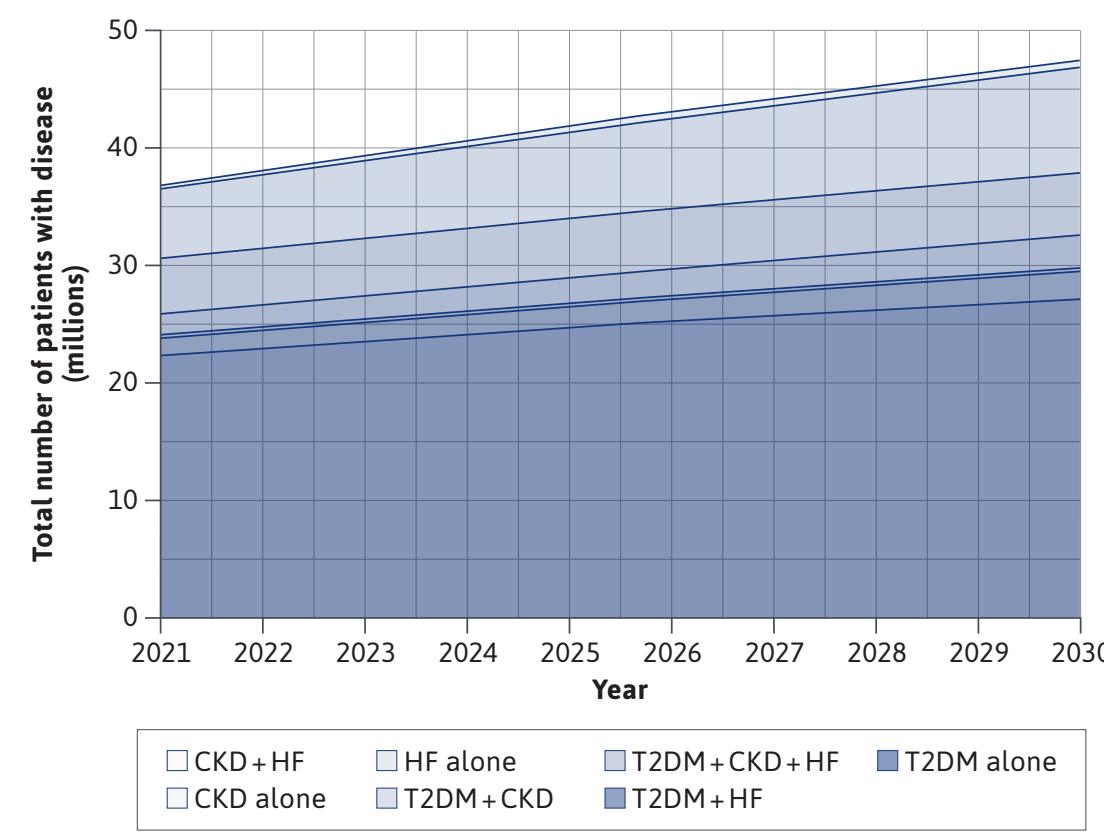

$C K D=$ chronic kidney disease; $H F=$ heart failure; T2DM = type 2 diabetes mellitus.

disease prevalence for single and multiple morbidities. An estimated 25.6, 8.1, and 6.8 million people are living with diagnosed T2DM, CKD, or HF, respectively, with 3.3 million living with 2 comorbidities, and approximately 200,000 with T2DM, CKD, and HF. The model estimated that total prevalence across all disease states will have increased by $28 \%$ in 2030 , with approximately 47 million people living with diagnosed cardiorenal disease (representing $17 \%$ of the adult population; Figure 2). Prevalence is predicted to increase across single health states and dual comorbid disease states, with $\mathrm{HF}$ and comorbid CKD having the largest percentage increase of $101 \%$ between 2021 and 2030.

The annual direct health care expenditure associated with cardiorenal disease is substantial. For 2021, the total costs attributed to cardiorenal disease estimated by our model are approximately $\$ 388$ billion, mostly comprised of hospital-related costs, which account for approximately $41 \%$, or $\$ 157$ billion, of the total. Patients with diagnosed HF or CKD account for $39 \%$ of the diagnosed cardiorenal population yet consume approximately $58 \%$ of total costs, or \$225 billion annually. Supplementary Figure 2 presents a stacked bar chart demonstrating the distribution of cost across the disease health states, with each segment representing the cost attributed to that disease state. Supplementary Figure 3 presents historic costs for the individual disease states and the projected costs predicted by the current analysis.
Across all disease areas, costs have risen substantially and are expected to continue to increase over the next decade.

Figure 3 illustrates the relationship between disease prevalence and proportion of total costs consumed by each health state. As demonstrated in the figure, patients with T2DM alone, while representing a greater percentage of the prevalent cardiorenal population (61\%) compared with other disease states, are associated with proportionally less annual health care expenditure at $\$ 156$ billion, or $40 \%$ of total costs. In contrast, patients diagnosed with CKD or HF consume disproportionally greater costs than those with other diseases; for example, those with T2DM+HF represent $5 \%$ of the total cardiorenal population but consume approximately $11 \%$ of total costs.

We estimate that the annual proportion of costs attributable to CKD or HF will increase from $54 \%$ (\$208 billion) in 2021 to $57 \%$ (\$286 billion) in 2030. Cumulatively, the direct health care cost of cardiorenal disease between 2021 and 2025 is estimated at $\$ 2.1$ trillion increasing to $\$ 4.5$ trillion in 2030 (Supplementary Figure 4A. Much of this cost burden is attributed to hospital-related events.

The total number of annual hospitalizations is estimated to increase from 6.2 to 7.7 million from 2021 to 2030. Patients with HF are subject to a high risk of hospitalization following diagnosis and, following an initial hospitalization, have a substantially higher lifetime risk of rehospitalization compared with the general population. Consequently, HF accounts for approximately $42 \%$ of the total number of hospitalizations. Supplementary Figure 4B illustrates the cumulative hospitalization expected to 2025 (32.6 million) and 2030 (69.4 million). 


\section{FIGURE 3 The Relationship Between Disease Prevalence and Cost in 2021}

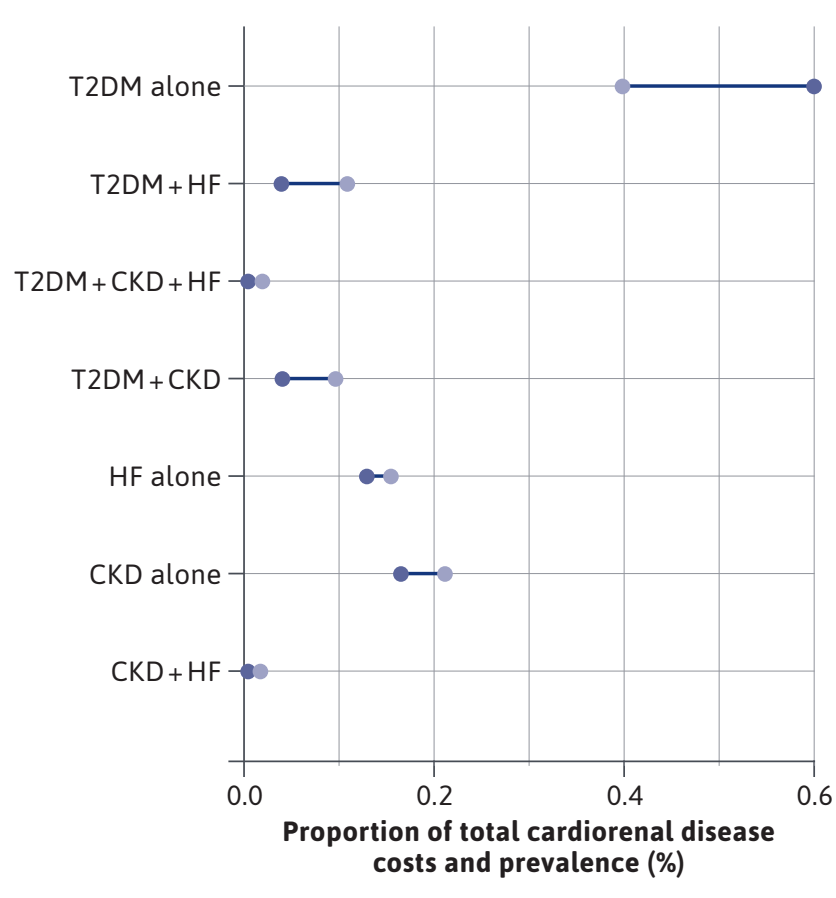

- Proportion of total cardiorenal disease costs - Proportion of total cardiorenal disease prevalence

$C K D=$ chronic kidney disease $\mathrm{HF}=$ heart failure; $T 2 D M=$ type 2 diabetes mellitus.

\section{THE POTENTIAL CONTRIBUTION OF NOVEL THERAPIES IN REDUCING THE US BURDEN OF CARDIORENAL DISEASE}

In considering therapeutic impact, and uptake for dapagliflozin, empagliflozin, canagliflozin, and sacubitril and valsartan, Figure 4 shows the potential reduction in total cumulative costs, hospitalization events and disease prevalence by the end of 2021, 2025 and 2030, respectively, compared to standard of care. Consistently, the expected reductions associated with dapagliflozin, expressed as a percentage, were greater than for other therapies reflecting a combination of broad trial generalizability and effectiveness applied across T2DM with or without HF/CKD, HF and CKD. Estimated cumulative costs to the end of 2021, 2025 , and 2030 were estimated to be $2.3 \%, 3.1 \%$, and $3.4 \%$ lower with dapagliflozin, compared with standard of care, driven by a combination of reduced event rates and disease prevalence. In absolute terms, this reflects a reduction in cumulative cost, to the end of 2021, 2025, and 2030, of $\$ 9$ billion, $\$ 64$ billion, and $\$ 153$ billion, respectively.

The cumulative number of hospitalizations to the end of 2021, 2025, and 2030 were estimated at 6.2, 32.7, and 69.4 million, respectively. Extrapolating the clinical trial evidence for dapagliflozin indicates that these could be reduced by $2.8 \%(173,000)$ in $2021,3.2 \%$ ( 1.1 million) by 2025 , and $3.4 \%$ (2.4 million) by 2030. Disease prevalence was estimated to be reduced by 208,500 by 2025 and 321,000 by 2030 with dapagliflozin.

\section{SENSITIVITY ANALYSIS}

As the presented analysis is dependent upon costs and resource use cited in the literature, a sensitivity analysis was conducted to evaluate the estimated reduction on costs and hospitalizations of considered therapies under alternative input assumptions. Costs and resource use in the model were varied by $20 \%$ above and below the mean value used in the analysis, whereas hazard ratios from the clinical trials were modeled using upper and lower confidence intervals. Supplementary Figures 5 and 6 present the expected cumulative percentage reduction in costs and hospitalizations by 2030, respectively, for each therapy. Under each considered scenario, all therapies remained beneficial, with dapagliflozin remaining as the therapy with the most benefit in terms of cost reduction and a reduced number of cumulative hospitalization events up to year 2030.

\section{Discussion}

T2DM, HF, and CKD are associated with significant levels of health care resource utilization, ${ }^{15,16}$ and as their prevalence continues to increase in the United States, ${ }^{17,18}$ so will their associated burden. We sought to integrate published evidence describing the burden of T2DM, HF, and CKD as individual discrete chronic conditions in order to consider the combined cardiorenal burden in the United States over the next decade. We validated prevalence projections for T2DM, ${ }^{19,20} \mathrm{CKD},{ }^{21} \mathrm{ESRD},{ }^{22}$ and $\mathrm{HF}^{3}$ with published data, and our findings are consistent with projections for each respective comorbidity.

With the emergence of novel therapies that provide cardio- and reno-protective benefits in those with and without diabetes, we have illustrated that there is value in considering a broader whole-disease approach to assessing disease burden and the value of therapeutic innovation. Our analysis has shown that extrapolating the results of recently reported cardiovascular outcomes trials for SGLT2is to a representative US population would likely 


\section{FIGURE 4 Expected Percentage Reductions Compared With Standard of Care in Direct Health Care Costs, Number of Hospitalizations, and Disease Prevalence Associated With Extrapolating Trial-Based Hazard Ratios for Each Respective Therapy When Considering the Generalizability of Trial Evidence}

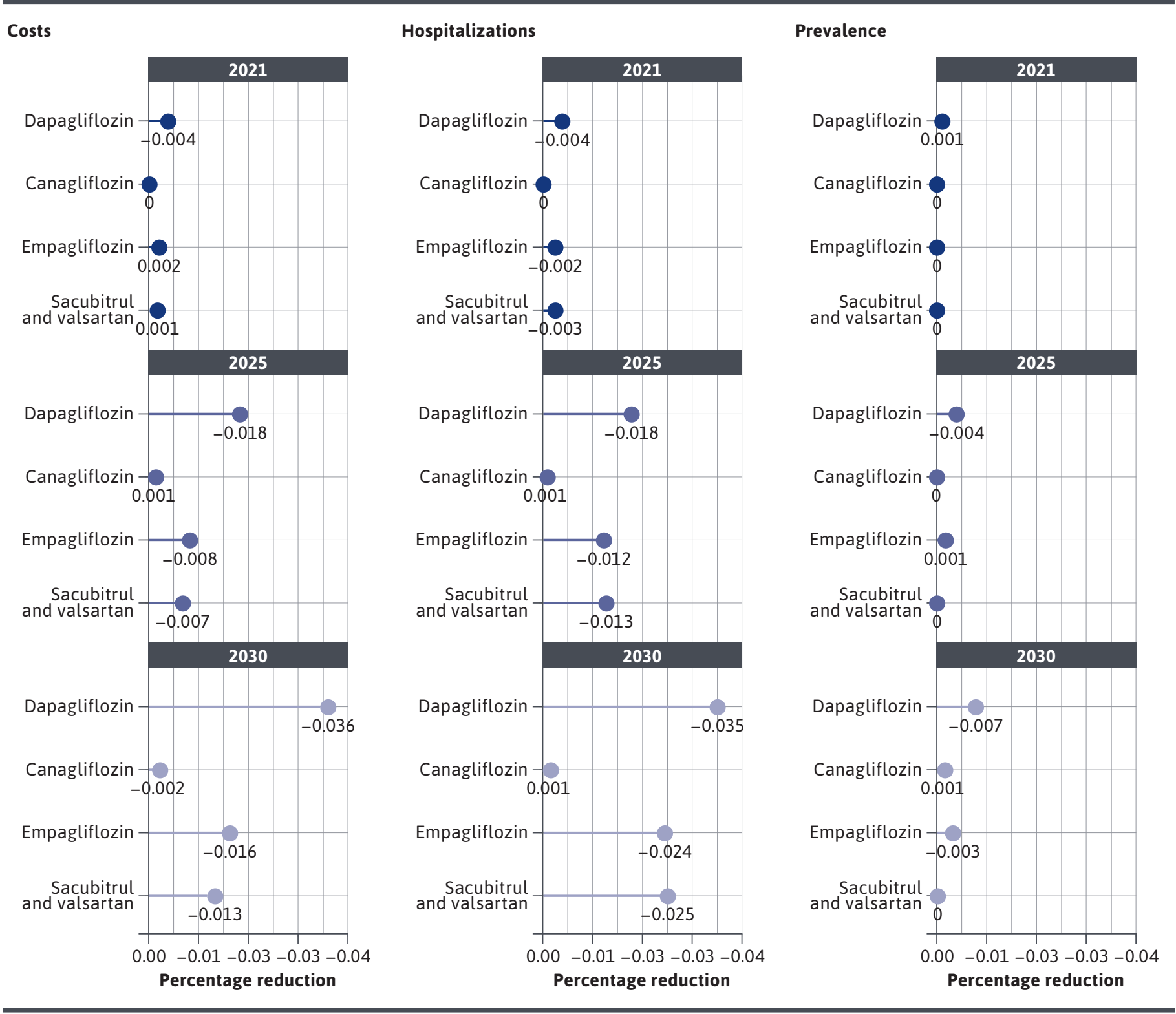

result in a substantial reduction in the number of hospitalizations, disease prevalence, and associated costs over the next decade.

These outcome benefits are likely to outweigh any adverse events associated with this therapeutic innovation. Indeed, even though SGLT2is have been associated with adverse effects related to their mechanism of action, including polyuria and intravascular volume depletion from osmotic diuresis and genitourinary infections from glycosuria, the clinical trials have demonstrated that SGLT2is are well tolerated, with a low risk of serious adverse effects such as ketoacidosis, lower limb amputations, and fractures. Assessing the risks; modifying antihypertensive, diuretic, and antiglycemic treatment; and only prescribing SGLT2is 
to suitable patients will help in maximizing the risk-benefit profile associated with this therapeutic innovation.

Recently, the fragility of the relationship between health care capacity and demand has been highlighted by the coronavirus disease 2019 pandemic with health care systems stretched despite unprecedented levels of additional funding. This is because system capacity is defined by more than bed availability, including factors such as staffing levels and access to investigations and therapeutic interventions. Consequently, economic evaluations should recognize that health care planning and decision making is undertaken by multiple stakeholders, often with differing, but not mutually exclusive, determinants of value. In recent years, the relationship between investment in health care and the rate of growth in the demand for health care services has focused attention on the concept of optimizing value, namely, maximizing the health outcomes achieved per population for unit of resource available. Although a CEA is frequently used in the valuation of therapeutic innovation, ${ }^{23}$ it can often be difficult to reconcile its output with the operational challenges facing the health care system. For example, where an intervention has multiple indications the assessment of value, via a CEA, is likely to underestimate the whole system impact of such a treatment. This is exemplified by the SGLT2is, where therapeutic indications extended beyond diabetes management, supported by large scale outcomes trials, into HF and CKD in people with and without diabetes. Based on such considerations, we undertook a holistic economic assessment that provides a broader perspective of the impact of therapy innovation, ie, SGLT2is.

\section{LIMITATIONS}

The current analysis has a number of limitations that must be taken into consideration when reviewing the data. Our assessment of the impact of therapeutic innovation on projected cardiorenal disease burden makes a number of strong assumptions. First, we applied trial-based hazard ratios to proportions of the comorbid population deemed representative of each trial. Our intention was to illustrate the potential impact of therapeutic innovation on disease burden without seeking to extrapolate beyond the evidence base. It is likely that prescribing patterns in clinical practice will differ significantly from the approach we have modeled, and, therefore, our projections should be viewed as "potential" benefits.

Second, we have restricted our analysis to those patients diagnosed with T2DM, HF, or CKD. The proportion of people with undiagnosed disease, particularly T2DM and CKD, in the United States is substantial. ${ }^{24-26}$ Consequently, the potential for broadening the impact of primary prevention within these populations is significant and has not been considered in this analysis.

Finally, it is noteworthy that real-world studies of SGLT2is in clinical practice have indicated that substantially greater benefits have been realized with their use than initial CEA suggested. For example, the CVD-REAL (Comparative Effectiveness of Cardiovascular Outcomes in New Users of SGLT-2 Inhibitors) study has reported clinically meaningful reductions in mortality, heart failure hospitalization, cardiovascular events, and kidney events suggesting the evidence from the SGLT2i clinical outcome studies translate robustly into clinical practice. ${ }^{27-29}$

It is likely that current modeling approaches underestimate the resource burden associated with hospitalization for HF and ESRD. Patients hospitalized for HF have a high incidence of readmission; studies have estimated 30-day readmission rates of $19 \%-27 \%^{30-32}$ and 5-year readmission rates of $80 \%{ }^{33}$ Frequent flyer cohorts (defined as patients who have been admitted at least 3 times in 12 months $^{34}$ ) have been observed and characterized within the HF population; in 1 study, $10 \%$ of incident HF patients had $\geq 10$ hospitalizations after their HF diagnosis, with the number of hospitalizations ranging from 0 to $42 .^{35}$ The prognosis for HF remains poor, with 5-year mortality rates estimated at $50 \%-60 \%{ }^{36,37}$ Several studies have shown that the resource use of HF patients increases substantially as they progress to end-of-life care ${ }^{38-40}$ with more than half the total costs in the last 2 years of life incurred in the final 6 months, $75 \%$ of which are related to hospitalization for HF. ${ }^{38}$ Similarly, current modeling approaches do not capture the resource burden of CKD progression and do not adequately capture the costs of ESRD. It is also important to recognize that many patients have multiple, interacting comorbidities.

Additional data and novel modeling approaches are required to capture the synergy between different conditions to describe outcomes more fully. Clarity on outcomes would ultimately improve estimation of the cost-effectiveness for drugs, such as dapagliflozin, that modify one or more of these comorbidities.

\section{Conclusions}

We have considered a holistic approach to characterizing the burden of cardiorenal disease that highlights both the current and future burden of T2DM, HF, and CKD in terms of disease incidence, prevalence, cost, and resource utilization. Our analyses demonstrate that considering HF and CKD risk prevention when managing patients with cardiorenal disease offers an opportunity to reduce this burden over the next 10 years in the US population. 


\section{DISCLOSURES}

This work was funded by AstraZeneca, which provided support for data analysis. McEwan, Morgan, and Boyce are employees of Health Economics and Outcomes Research Ltd., Cardiff, UK, which received fees from AstraZeneca in relation to this study. Song and Huang are employees of AstraZeneca. Bergenheim is an employee of AstraZeneca and holds AstraZeneca stocks/stock options. Green has no conflicts of interest to declare.

\section{ACKNOWLEDGMENTS}

Angharad R Morgan provided medical writing support, which was funded by AstraZeneca.

\section{REFERENCES}

1. Briggs A, Claxton K, Sculpher M. Decision modelling for health economic evaluation. Oxford University Press; 2006.

2. Savira F, Magaye R, Liew D, et al. Cardiorenal syndrome: Multi-organ dysfunction involving the heart, kidney and vasculature. $\mathrm{Br}$ J Pharmacol. 2020;177(13):2906-22.

3. Heidenreich PA, Albert NM, Allen LA, et al. Forecasting the impact of heart failure in the United States: a policy statement from the American Heart Association. Circ Heart Fail. 2013;6(3):606-19.

4. Saran R, Robinson B, Abbott KC, et al. US Renal Data System 2019 Annual Data Report: Epidemiology of Kidney Disease in the United States. Am J Kidney Dis. 2020;75(1 Suppl 1):A6-7.

5. McEwan P, Bennett H, Khunti K, et al. Assessing the cost-effectiveness of sodium-glucose cotransporter-2 inhibitors in type 2 diabetes mellitus: a comprehensive economic evaluation using clinical trial and real-world evidence. Diabetes Obes Metab. 2020;22(12):2364-74.

6. McEwan P, Morgan AR, Boyce R, et al. The cost-effectiveness of dapagliflozin in treating high-risk patients with type 2 diabetes mellitus: an economic evaluation using data from the DECLARETIMI 58 trial. Diabetes Obes Metab. 2021;23(4):1020-09.
7. McEwan P, Darlington O, McMurray JJV, et al. Cost-effectiveness of dapagliflozin as a treatment for heart failure with reduced ejection fraction: a multinational health-economic analysis of DAPA-HF. Eur J Heart Fail. 2020;22(11):2147-56.

8. McEwan P, Darlington O, Wheeler D, et al. POS-335 Cost-effectiveness of dapagliflozin as a treatment for chronic kidney disease: a health-economic analysis of DAPA-CKD. Kidney International Reports. 2021;6(4):S14546.

9. United States Census Bureau. Table S0101: age and sex. Accessed July 28, 2021. https://data.census.gov/cedsci/table?q=population\%20gender\&h idePreview=false\&tid=ACSST1Y2012. $\underline{\text { S0101\&vintage }=2018}$

10. United States Bureau of Labor Statistics. Databases, tables \& calculators by subject. Accessed July 28, 2021. https://data.bls.gov/timeseries/ CUUR0000SAM?output view $=$ pct $12 \mathrm{mths}$

11. Wiviott SD, Raz I, Bonaca MP, et al. Dapagliflozin and cardiovascular outcomes in type 2 diabetes. N Engl J Med. 2019;380(4):347-57.

12. Zinman B, Wanner C, Lachin JM, et al. Empagliflozin, cardiovascular outcomes, and mortality in type 2 diabetes. $\mathrm{N}$ Engl J Med. 2015;373(22):2117-28.

13. Neal B, Perkovic V, Mahaffey KW, et al. Canagliflozin and Cardiovascular and Renal Events in Type 2 Diabetes. N Engl J Med. 2017;377(7):644-57.

14. Mosenzon O, Wiviott SD, Cahn A, et al. Effects of dapagliflozin on development and progression of kidney disease in patients with type 2 diabetes: an analysis from the DECLARE-TIMI 58 randomised trial. Lancet Diabetes Endocrinol. 2019;7(8):606-17.

15. Annavarapu S, Ghosh S, Li Y, et al. Health care resource utilization among patients with T2D and cardiovascular-, heart failure-, or renal-related hospitalizations. Am J Manag Care. 2020;26(6):e166-71.
16. Folkerts K, Petruski-Ivleva N, Kelly A, et al. Annual health care resource utilization and cost among type 2 diabetes patients with newly recognized chronic kidney disease within a large U.S. administrative claims database. J Manag Care Spec Pharm. 2020;26(12):1506-16. doi:10.18553/jmcp.2020.26.12.1506

17. Arnold SV, Kosiborod M, Wang J, et al. Burden of cardio-renal-metabolic conditions in adults with type 2 diabetes within the Diabetes Collaborative Registry. Diabetes Obes Metab. 2018;20(8):2000-03.

18. Ford ES, Giles WH, Mokdad AH. Increasing prevalence of the metabolic syndrome among U.S. adults. Diabetes Care. 2004;27(10):24444-9.

19. International Diabetes Federation. IDF Diabetes Atlas. 10th Edition. Accessed July 28, 2021. https://diabetesatlas.org/data/ en/country/211/us.html

20. Lin J, Thompson TJ, Cheng YJ, et al. Projection of the future diabetes burden in the United States through 2060. Popul Health Metr. 2018;16(1):9.

21. Centers for Disease Control and Prevention. Chronic kidney disease (CKD) surveillance system. Accessed July 28, 2021. https://nccd.cdc.gov/CKD/detail. aspx?Qnum=Q677\#refreshPosition

22. McCullough KP, Morgenstern H, Saran R, et al. Projecting ESRD Incidence and Prevalence in the United States through 2030. J Am Soc Nephrol. 2019;30(1):127-35.

23. Parizo JT, Goldhaber-Fiebert JD, Salomon JA, et al. Cost-effectiveness of dapagliflozin for treatment of patients with heart failure with reduced ejection fraction. JAMA Cardiol. 2021;6(8):926-35.

24. Beagley J, Guariguata L, Weil C, et al. Global estimates of undiagnosed diabetes in adults. Diabetes Res Clin Pract. 2014;103(2):150-60.

25. Cowie CC, Rust KF, Ford ES, et al. Full accounting of diabetes and prediabetes in the U.S. population in 1988-1994 and 2005-2006. Diabetes Care. 2009;32(2):287-94. 
26. Plantinga LC, Crews DC, Coresh J, et al. Prevalence of chronic kidney disease in US adults with undiagnosed diabetes or prediabetes. Clin J Am Soc Nephrol. 2010;5(4):673-82.

27. Kosiborod M, Cavender MA, Fu AZ, et al. Lower risk of heart failure and death in patients initiated on sodium-glucose cotransporter-2 inhibitors versus other glucose-lowering drugs: the CVD-REAL study (Comparative Effectiveness of Cardiovascular Outcomes in New Users of Sodium-Glucose Cotransporter-2 Inhibitors). Circulation. 2017;136(3):249-59.

28. Kosiborod M, Lam CSP, Kohsaka S, et al. Cardiovascular events associated with SGLT-2 inhibitors versus other glucose-lowering drugs: the CVD-REAL 2 study. J Am Coll Cardiol. 2018;71(23): 2628-39.

29. Heerspink HJL, Karasik A, Thuresson M, et al. Kidney outcomes associated with use of SGLT2 inhibitors in real-world clinical practice (CVDREAL 3): a multinational observational cohort study. Lancet Diabetes Endocrinol. 2020;8(1):27-35.

30. Bueno H, Ross JS, Wang Y, et al. Trends in length of stay and short-term outcomes among Medicare patients hospitalized for heart failure, 1993-2006. JAMA. 2010;303(21):2141-47.

31. Gheorghiade M, Vaduganathan $\mathrm{M}$, Fonarow GC, et al. Rehospitalization for heart failure: problems and perspectives. J Am Coll Cardiol. 2013;61(4):391-403.
32. Ziaeian B, Hernandez AF, DeVore AD, et al. Long-term outcomes for heart failure patients with and without diabetes: From the Get With the Guidelines-Heart Failure Registry. Am Heart J. 2019;211:1-10.

33. Shah KS, Xu H, Matsouaka RA, et al. Heart failure with preserved, borderline, and reduced ejection fraction: 5-year outcomes. J Am Coll Cardiol. 2017;70(20):2476-86.

34. Williams S, Bottle A, Rogers R, et al. "Frequent flier" patients. BMJ. 2005;330(7496):869.

35. Dunlay SM, Redfield MM, Weston SA, et al. Hospitalizations after heart failure diagnosis a community perspective. J Am Coll Cardiol. 2009;54(18):1695-702.

36. Ziaeian B, Fonarow GC. Epidemiology and aetiology of heart failure. Nat Rev Cardiol. 2016;13(6):368-78.

37. Roger VL. Epidemiology of heart failure. Circ Res. 2013;113(6):646-59.

38. Russo MJ, Gelijns AC, Stevenson LW, et al. The cost of medical management in advanced heart failure during the final two years of life. J Card Fail. 2008;14(8):651-58.

39. Chun S, Tu JV, Wijeysundera HC, et al. Lifetime analysis of hospitalizations and survival of patients newly admitted with heart failure. Circ Heart Fail. 2012;5(4):414-21.

40. Desai AS, Stevenson LW. Rehospitalization for heart failure: predict or prevent? Circulation. 2012;126(4):501-06.
41. Wittbrodt ET, Eudicone JM, Bell KF, et al. Eligibility varies among the 4 sodium-glucose cotransporter-2 inhibitor cardiovascular outcomes trials: implications for the general type 2 diabetes US population. Am J Manag Care. 2018;24 (8 Suppl):S138-45.

42. Heerspink HJL, Stefánsson BV, Correa-Rotter R, et al. Dapagliflozin in patients with chronic kidney disease. N Engl J Med. 2020;383(15):1436-46.

43. McMurray JJV, Solomon SD, Inzucchi SE, et al. Dapagliflozin in Patients with heart failure and reduced ejection fraction. N Engl J Med. 2019;381(21):1995-2008.

44. Wanner C, Inzucchi SE, Lachin JM, et al. Empagliflozin and progression of kidney disease in type 2 diabetes. $\mathrm{N}$ Engl J Med. 2016;375(4):323-34.

45. Packer M, Anker SD, Butler J, et al. Cardiovascular and renal outcomes with empagliflozin in heart failure. N Engl J Med. 2020;383(15):1413-24.

46. Perkovic V, de Zeeuw D, Mahaffey KW, et al. Canagliflozin and renal outcomes in type 2 diabetes: results from the CANVAS Program randomised clinical trials. Lancet Diabetes Endocrinol. 2018;6(9):691-704.

47. McMurray JJ, Packer M, Desai AS, et al. Angiotensin-neprilysin inhibition versus enalapril in heart failure. $\mathrm{N}$ Engl J Med. 2014;371(11):993-1004. 